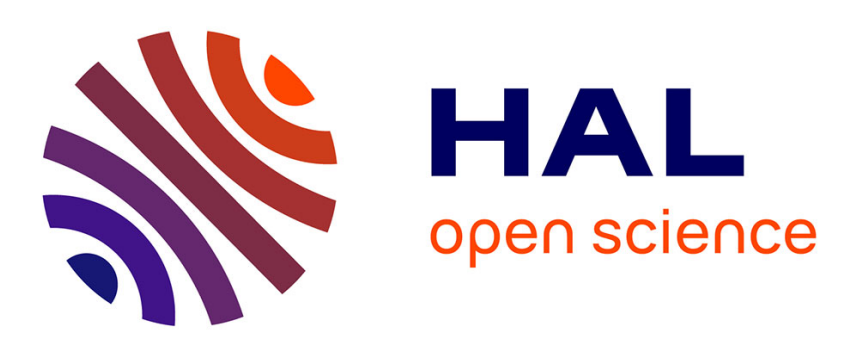

\title{
Historique de l'évolution du cadre législatif et réglementairedes modes de ventes des bois des forêts publiques
}

\author{
Alfred Estrade, Georges-André Morin
}

\section{To cite this version:}

Alfred Estrade, Georges-André Morin. Historique de l'évolution du cadre législatif et réglementairedes modes de ventes des bois des forêts publiques. Revue forestière française, 2006, 58 (3), pp.245-256. 10.4267/2042/5997 . hal-03449368

\section{HAL Id: hal-03449368 \\ https://hal.science/hal-03449368}

Submitted on 25 Nov 2021

HAL is a multi-disciplinary open access archive for the deposit and dissemination of scientific research documents, whether they are published or not. The documents may come from teaching and research institutions in France or abroad, or from public or private research centers.
L'archive ouverte pluridisciplinaire HAL, est destinée au dépôt et à la diffusion de documents scientifiques de niveau recherche, publiés ou non, émanant des établissements d'enseignement et de recherche français ou étrangers, des laboratoires publics ou privés. 


\section{HISTOIRE ET TERAITOIRES}

\section{HISTORIQUE DE L'ÉVOLUTION \\ DU CADRE LÉGISLATIF ET RÉGLEMENTAIRE \\ DES MODES DE VENTES DES BOIS \\ DES FORÊTS PUBLIQUES}

\section{Alfred Estrade - Georges-André Morin}

La loi n 2005-157 du 23 février 2005 "relative au développement des territoires ruraux » (LDTR) est un texte abondant. La forêt couvrant aujourd'hui plus de $30 \%$ de l'espace rural, dont un peu plus du quart appartient à l'État et aux collectivités locales, le gestionnaire des forêts publiques, l'Office national des Forêts (ONF), est concerné par son article 227-III modifiant les dispositions du Code forestier relatives aux modalités des ventes de bois des forêts publiques.

III. Les articles L.134-1 et L.134-7 du même code sont ainsi rédigés :

"Art. L.134-1. - Toute vente doit être conforme aux dispositions de l'article L.134-7 et des règlements pris pour son application, à peine de nullité ».

"Art. L.134-7. - Les coupes et produits des coupes dans les bois et forêts de l'État sont vendus par l'Office national des Forêts soit par adjudication ou appel d'offres, soit de gré à gré, dans des conditions fixées par décret en Conseil d’État ».

«Des contrats d'approvisionnement pluriannuels peuvent être conclus ».

Votée conforme au projet de loi dès la première lecture, tant devant l'Assemblée nationale que devant le Sénat, cette disposition met un terme à de longs tâtonnements. Depuis 1969, c'est la quatrième réforme des articles du Code forestier relatifs aux modalités des ventes de bois des forêts publiques, restées pratiquement inchangées depuis l'Ordonnance du 16 août 1669. Dans la seconde moitié du XXe siècle, le contexte économique des activités d'exploitation forestière en France est radicalement différent de ce qu'il était au XVII ${ }^{\mathrm{e}}$ siècle. Les extensions forestières permises par les progrès de l'agriculture et l'exode rural ainsi que l'évolution de la sylviculture ont considérablement augmenté les potentialités de la forêt française. Le Fonds forestier national, créé en 1946 sur une recommandation du "plan Monnet", a accéléré cette progression. Les secteurs de l'exploitation forestière et de la scierie ont évolué d'un artisanat traditionnel à l'ère industrielle. Les industries du bois, fortement capitalistiques, recherchent en contractualisant leurs approvisionnements une sécurité en quantité et en qualité, et une stabilité des prix.

La publication, au Journal officiel du 23 novembre 2005, du décret d'application n 2005-1445 parachève l'évolution de ces textes, ce qui offre l'occasion de faire un point. 
Alfred Estrade - Georges-André Morin

\section{DE 1669 À 1926 : L’ADJUDICATION PUBLIQUE, MODE DE VENTE EXCLUSIF}

\section{L’Ordonnance des “Eaux et Forêts” du 16 août 1669}

Cette ordonnance constitue un bon point de départ, sans reprendre tout l'historique antérieur. Le début du règne personnel de Louis XIV est celui d'une stabilité retrouvée. La gestion des forêts de la Couronne est alors importante. Leur apport aux recettes de l'État n'est pas à négliger. Surtout, les ambitions maritimes de la France font du bois un matériau stratégique, tant pour la marine de guerre que pour celle de commerce.

Les désordres du temps n'avaient pas épargné le corps forestier. Jean Orieux (1976) écrit dans sa biographie de Jean de La Fontaine, (issu d'une dynastie de forestiers qui remonte peut-être à un grand maître du XIV siècle), que celui-ci " passait pour arrondir ses émoluments, prélevant du bois de feu à peu près sans contrôle. Il avait droit à une dîme et quelques coupes faites à son profit : il ne s'en priva pas ", dans un contexte où " chacun fraudait de son mieux pour compenser la modestie de la rémunération ducale » ${ }^{(1)}$.

Le texte de l'ordonnance reprenait, réordonnait et complétait des textes antérieurs. Le titre XV traite des ventes. Constitué de 52 articles, c'est le plus important de l'ordonnance. Son article $1^{\text {er }}$ : "Il ne sera fait aucune vente dans Nos forêts, bois et buissons, soit de futaie ou de taillis, que suivant le Règlement qui en aura été arrêté en notre Conseil " institue l'obligation d'un règlement des coupes, approuvé par l'autorité royale qui encadre leur pratique, désormais soumise à programmation et approbation. L'article 2 fixe les modalités des ventes : "Les adjudications des ventes de Nos bois tant en futaie qu'en taillis, ne pourront être faites à l'advenir que par les Grands Maîtres, faisant défense aux officiers des Maîtrises de reconnaître d'autres personnes, à peine d'en répondre en leur nom ».

Contrairement à une idée reçue, la Révolution, si elle suscite de considérables transferts de propriété, ne bouleverse pas la législation forestière. L'article 10 de la loi du 16 nivôse an IX (6 janvier 1801) " relative à l'organisation d'une nouvelle administration forestière " rappelle que "Toutes dispositions des lois et règlement sur le régime forestier, auxquelles il n'est pas dérogé par la présente, continueront d'être exécutées jusqu'à ce qu'il en ait été autrement ordonné ». L'administration est réorganisée, mais dans le cadre de l'ordonnance de 1669. L'arrêté du 22 frimaire an XI complète le texte de 1669, en « fixant les termes et modes de paiements du prix des adjudications des coupes ordinaires de bois ».

\section{LE CODE FORESTIER DE 1827}

Ce code (voir publication commentée de Coin-Delisle et Frederich, 1827-28) a été adopté par les chambres le 21 mai 1827 et promulgué par Charles X le 31 juillet. Le projet fut présenté par Martignac, alors directeur général de l'Enregistrement et des Domaines (il fut nommé président du Conseil en janvier 1828). Sa présentation devant la Chambre des Députés, le 26 décembre 1826, est dans le droit-fil de la continuité. "On a conservé dans l'ensemble des mesures adoptées ce que l'ordonnance de 1669 contenait de bon, d'utile et d'éprouvé, et on y a ajouté ce que l'expérience a fait juger propre à remédier aux inconvénients reconnus ».

S'agissant des coupes, il déclare : "Les coupes dont l'aménagement permet l'exploitation doivent être adjugées. Ces coupes sont une branche importante des revenus publics : il était du

(1) Extrait des pages 79 et 81 de ladite biographie ; il s'agit des forêts du duc de Bouillon. 
plus grand intérêt de les placer à l'abri de la fraude, de la connivence et même de l'erreur... les mesures les plus sévères sont prises pour assurer la publicité des adjudications, la concurrence et la liberté des enchères ». Déclaration reprise devant la Chambre des Pairs (2) : "Les coupes qui sont arrivées à leur maturité, ainsi que les fruits des forêts connus sous le nom de glandée et de panage, doivent être soumis à une adjudication ; ces branches importantes du revenu public doivent être sagement garanties de la fraude et de l'erreur. L'expérience a dévoilé à cet égard des dangers nombreux contre lesquels la loi doit armer l'administration. Des mesures combinées avec soin vous paraîtront propres à assurer d'une part la publicité des adjudications, la concurrence et la liberté des enchères, de l'autre, l'ordre, la régularité, la juste limite des exploitations, et la répression des abus dont les adjudications peuvent toujours être la source ».

Le Code sépare les dispositions relatives à la gestion des forêts et à la définition de l'assiette des coupes, articles 15 et 16 , de celles relatives aux ventes, articles 17 et suivants. L'innovation fondamentale par rapport à l'ordonnance de 1669 est l'explicitation de la notion d'aménagement à l'article 15 qui, dépassant celle de règlement de coupe, introduit une notion de gestion planifiée de la forêt, prise sous tous ses aspects ${ }^{(3)}$.

Le principe de l'obligation du recours à la vente par adjudication est posé à l'article 17 : "Aucune vente ordinaire ou extraordinaire ne pourra avoir lieu dans les bois et forêts de l'État que par voie d'adjudication publique, laquelle devra être annoncée au moins quinze jours à l'avance, par des affiches apposées dans le chef-lieu du département, dans le lieu de la vente, dans la commune de situation des bois, et dans les communes environnantes ${ }^{(4)} »$.

Ensuite, l'article 18 précise que " toute vente autrement que par adjudication sera considérée comme vente clandestine et déclarée nulle " et l'article 19 prévoit la nullité de la vente si la publicité a été insuffisante : "Sera de même annulée, quoique faite par adjudication publique, toute vente qui n'aura point été précédée des publications et affiches prescrites à l'art. 17, ou qui aura été effectuée dans d'autres lieux ou à un autre jour que ceux qui ont été indiqués dans les affiches ou le procès-verbal de remise de vente $"{ }^{(5)}$. Enfin, l'article 20 traite des contestations survenant lors des adjudications. Ces dispositions législatives sont complétées par les articles 73 à 85 de l'ordonnance royale prise pour leur application.

\section{LA LÉGALISATION DES VENTES AMIABLES : UNE LONGUE TRANSITION DEPUIS 1926}

\section{En 1926, l'introduction des ventes amiables}

En 1926, il est introduit un nouvel article 20 qui prévoit à titre dérogatoire la possibilité de ventes amiables :

«Par dérogation aux articles 17, 18, et 19 du présent code, le ministre de l'agriculture peut autoriser, dans les forêts soumises au régime forestier, la cession de vente amiable de bois abattus

(2) Le rapport de la commission de la Chambre des Pairs pose le cadre général de la gestion d'un bien dont l'État est propriétaire, justification de mesures spécifiques mais rappel des principes de l'exercice du droit de propriété. "L'État est propriétaire du domaine public; or il appartient au propriétaire de prescrire les règles de l'administration de son domaine, et la loi est l'expression de la volonté de l'État. C'est pour cette raison, sans doute, que dans tous les temps, la législation a agi sur le domaine de l'État et sur son administration ».

(3) L'exposé des motifs, rédigé en 1826, présente l'ensemble des fonctions de la forêt : "Ce n'est pas seulement par les richesses qu'offre l'exploitation des forêts sagement combinée qu'il faut juger de leur utilité ; leur existence même est un bienfait inappréciable pour les pays qui les possèdent; soit qu'elles protègent et alimentent les sources des rivières, soit qu'elles soutiennent et raffermissent le sol des montagnes, soit qu'elles exercent sur l'atmosphère une heureuse et salutaire influence ". C'est même écrit en français, sans recours au jargon anglicisé des "aménités " et autres "produits non-marchands ».

(4) Le débat a porté sur la notion de "commune environnante " qu'il fallait entendre par " commune limitrophe ».

(5) Il fut relevé à la Chambre des Pairs que l'usage prévoit également l'indication de l'heure ; l'article 84 de l'ordonnance précisa

"les affiches indiquent le lieu, le jour et l'heure ". 
Alfred Estrade - Georges-André Morin

ou façonnés, de bois incendiés, de chablis ou bois dépérissants qui sont restés invendus en adjudication publique ou de bois qui, en raison soit de leur situation, soit de leur faible valeur ne sont pas susceptibles d'être vendus en adjudication publique. Le prix de cession est fixé par le ministre de l'agriculture ou son délégué ».

Dans son ouvrage “Législation et politique forestières", l'ingénieur général Meyer qualifie cette modification d'" importante dérogation », soulignant que " pour toutes ces catégories de bois, l'adjudication obligatoire se révélant infructueuse ou impossible, la cession amiable permet de tirer le meilleur parti des bois et d'éviter leur dépréciation totale » (Meyer, 1968).

\section{L’article 16 de la loi de finances rectificative 69-1160 du 24 décembre 1969}

L'ONF avait été créé par l'article $1^{\mathrm{er}}$ de la loi de finances rectificative pour 1964 (loi 64-1278 du 23 décembre 1964) dont l'exposé des motifs précise : "C'est une nécessité pour la Nation d'adapter sa production forestière à des besoins sans cesse croissants. Pour y parvenir, le Parlement a déjà adopté en 1963 une loi permettant d'améliorer la production des forêts privées. Il reste à porter à son maximum la production des forêts de l'État et des autres collectivités publiques dont l'ensemble constitue un patrimoine de plus de quatre millions d'hectares, l'unité de gestion forestière la plus vaste et la plus riche en possibilités d'avenir de la Communauté économique européenne ».

Cependant, l'adoption de cet article avait suscité un difficile débat parlementaire, tant en termes de procédure - il fut reproché au Gouvernement d'avoir procédé par un simple article de loi de finances - que sur le fond. Trois freins au développement du nouvel établissement furent introduits, les deux premiers proviennent d'un amendement gouvernemental de compromis se substituant à un certain nombre d'amendements parlementaires, le troisième résulte d'un amendement parlementaire voté contre l'avis du Gouvernement :

- "l'ONF ne pourra, dans le cadre de sa mission, ni étendre ses activités d'exploitation en régie au-delà de celles qui sont actuellement assurées par l'administration des eaux et forêts,

- ni entreprendre une activité nouvelle, sauf autorisation expresse et préalable du ministre de l'agriculture et dans la mesure où l'initiative privée ne permettrait pas de satisfaire les besoins,

- il ne participe ni directement ni indirectement à des entreprises commerciales ou industrielles, quel que soit leur objet».

Le préfet Delaballe, concepteur de la réforme et premier directeur général de l'ONF, en souligne le conservatisme : "Trois amendements donc, manifestement négatifs... il faut bien en prendre acte. Une loi sans doute n'est pas figée, et ce qu'un législateur a fait, un autre peut le défaire ou l'améliorer » (Delaballe, s.d.). Le nouvel établissement perdait la possibilité juridique de développer la vente de bois façonnés dont disposait l'ancienne Direction générale des Eaux et Forêts. Sous son impulsion, il est procédé, dès 1969, à une première réforme des articles 17 à 20 du Code forestier en élargissant la possibilité de ventes amiables. "Il ne peut être procédé à des ventes à l'amiable, par dérogation à l'article 17 ci-dessus, que pour des motifs impérieux d'ordre technique ou commercial dans des cas définis par décret en Conseil d'État. Ces ventes sont soumises à l'approbation préalable de l'autorité supérieure ".

Même si dans le principe on reste dans le domaine du dérogatoire, cette disposition est à l'évidence beaucoup plus large que celle de 1926.

Le décret d'application du 12 mars 1973 prévoit huit cas d'autorisation de la vente amiable. Là encore, l'exposé des motifs est clair et sans ambiguïté : "Enfin, l'autorisation de vendre à l'amiable serait ouverte dans des cas où des motifs purement commerciaux le justifient ; ce sont 
les paragraphes 5 et 6 de l'article 16 qui visent essentiellement les situations suivantes :

- cas de quasi-monopole de fait,

- cas où le vendeur a besoin de susciter un débouché régulier de ses produits.

Bien que ces deux cas restent assez prudemment limités, ils ouvrent une possibilité d'action commerciale nouvelle qui correspond bien aux intentions du législateur lorsqu'il a créé l'Office sous la forme d'un établissement industriel et commercial ».

En 1979, ces dispositions sont codifiées aux articles L.134-7, L.134-8 et R.134-16 du Code forestier.

La même année, le rapport confié par les ministres de l'Agriculture et de l'Industrie à MM. Jean Méo, ingénieur des mines, ancien conseiller du général de Gaulle pour les affaires économiques, et Yves Bétolaud, ingénieur général du génie rural, des eaux et des forêts, alors directeur général de l'ONF, sur le devenir de la forêt française reprenait ce point dans ses conclusions. " ... Avec prudence et détermination, il faut développer des modes de vente introduits au cours des dernières années mais dont l'extension est encore limitée : prévente, vente de bois façonnés, vente de gré à gré non publique, avec appel d'offres ou avec un seul acheteur, contrats pluriannuels... " (Bétolaud et Méo, publié en 1980).

Malgré la volonté des deux premiers directeurs généraux de l'Office, MM. Delaballe et Bétolaud, ces dispositions restent sans utilisation effective. En effet, parmi les cas énumérés dans le décret, le $6^{\circ}$ était rédigé "pour assurer l'écoulement régulier des produits de la forêt ", ce qui ouvrait, sous le contrôle du ministère de tutelle, une large possibilité de développement des ventes amiables. Mais une vente amiable supposant deux contractants, l'ONF estimait que la formulation "l'écoulement régulier des produits de la forêt " ne justifiait pas un contrat dont la finalité serait l'approvisionnement régulier d'une industrie.

Une application est pourtant faite en 1986, sous l'impulsion de René Souchon ministre chargé de la Forêt, en vue de créer une importante scierie industrielle à Sougy dans la Nièvre. La position du ministre et son interprétation "biunivoque" du $6^{\circ}$ du décret de 1973 est confirmée par une note d'analyse établie par M. Denoix de Saint-Marc rapporteur en 1973 du projet de décret devant le Conseil d'État ${ }^{(6)}$. Le contrat (mais pas l'autorisation ministérielle de sa passation) fut l'objet d'un recours devant le tribunal administratif de Paris intenté par un GIE de scieurs constitué pour les besoins de la cause. Les requérants sont déboutés sur un motif de compétence. Le Conseil d'État confirme cette décision par un arrêt qui reprend, au cas de l'ONF, la règle générale selon laquelle les contentieux relatifs à l'activité commerciale d'un établissement public ressortissent aux juridictions judiciaires. L'ONF considéra cependant que ce succès formel ne constituait pas une base suffisante pour développer les contrats, malgré le succès technique et économique de la scierie de Sougy.

\section{La loi 91-5 du 3 janvier 1991, « modifiant diverses dispositions intéressant l'agriculture et la forêt "}

L'article 17 de cette loi modifie de nouveau l'article L.134-8 relatif aux ventes amiables :

"Il ne peut être procédé à des ventes à l'amiable par dérogation à l'article L.134-7 ci-dessus, que pour des motifs d'ordre technique ou commercial dans des cas et selon les modalités définis par décret en Conseil d'État».

(6) Par ailleurs, une note remise au ministre par un cabinet d'avocats spécialistes du droit de la concurrence souligne que les textes de 1969 et 1973 introduisent un large pouvoir d'appréciation économique de la situation du marché et que "les auteurs du règlement ont ainsi clairement voulu permettre une exploitation plus rationnelle de la forêt, donnant aux utilisateurs de bois une sécurité d'approvisionnement exigée des techniques industrielles et que permet difficilement une seule procédure d'adjudication publique ". 
Par rapport au texte antérieur, deux modifications de détail : la suppression de l'adjectif « impérieux » et l'introduction " des modalités » de la vente. La suppression de l'autorisation de la tutelle est plus importante. Un EPIC doit pouvoir faire un acte de gestion courante sans en référer à sa tutelle.

En fait, l'ingénieur général Touzet, alors directeur général de l'ONF, en convient dans un courrier du 25 mars $2003^{(7)}$, il s'agissait "d'un toilettage de ces textes, inutile au point de vue du juriste, utile pour museler les oppositions ». Dans le même courrier, il souligne "au moins en 1991... des groupes de pression aussi bien intérieurs à l'ONF, les syndicats, mais pas uniquement, qu'extérieurs, étaient opposés aux ventes de gré à gré. Les raisons de ces différents groupes étant d'ailleurs différentes ». Il souligne que cet article procède d'un amendement parlementaire suscité par le Gouvernement, mais qu'il ne figurait pas dans le projet de loi transmis au Conseil d'État qui, selon M. Touzet, "n'aurait pas manqué d'en souligner l'inutilité totale ». Puis le décret du 27 mars 1993 réécrit, toujours dans l'esprit "variations sur un thème ", l'article R.134-16, substituant une rédaction plus détaillée à son $6^{\circ}$.

\section{La loi d'orientation sur la forêt du 9 juillet 2001}

La précédente modification n'ayant entraîné aucune évolution, la loi d'orientation sur la forêt (LOF) du 9 juillet 2001 comporte un nouvel ajustement, dans la ligne du précédent : son article 11-III. La section du code relative aux ventes amiables devient "procédures de vente", et les deux alinéas suivants sont introduits à l'article L.134-7 :

"Il peut être aussi procédé à des ventes de gré à gré pour des motifs d'ordre technique ou commercial ou économique dans des cas et selon les modalités définis par décret en Conseil d'État.

Des contrats d'approvisionnement pluriannuels peuvent être conclus ».

Le premier de ces alinéas est, à deux mots près, la reprise de la rédaction antérieure de l'article L.134-8 relatif aux ventes amiables. Désormais les ventes amiables (devenues de gré à gré) ne sont plus dérogatoires : "il peut être aussi procédé ».

Par voie de conséquence, la section III "ventes à l'amiable", constituée du seul article L.134-8 désormais repris à l'article précédent, est supprimée (cf. article 72 de la LOF) mais cette modification de coordination s'avère incomplète.

Le rapporteur devant l'Assemblée nationale précise que "les modes de vente de l'ONF n'étaient plus adaptés aux exigences économiques modernes ", que "le mode de vente normal impliquant publicité et appel à la concurrence... entraînait des contraintes très lourdes pour les acheteurs, astreints à la reconnaissance préalable d'un grand nombre de coupes ", et il considère que «la loi permet désormais un recours plus large aux ventes de gré à gré dès lors qu'un motif d'ordre technique, commercial ou économique le justifie ».

La seule modification effective, en dehors du changement de la place de cette disposition dans le Code forestier, est donc le rajout du motif " économique " aux motifs "technique et commercial » déjà introduits en 1969, mais rien dans l'exposé des motifs, ni dans les rapports des assemblées, ni dans les débats, ne permet d'en expliquer la portée.

Étrange utilisation de la loi où, à trois reprises, on réécrit la forme d'un texte pour tenter de l'appliquer ! Même si le Parlement n'a fait que revoter l'existant, la redondance des textes

(7) Ce courrier nous avait été adressé dans le cadre de la mission sur les fruitières de Franche-Comté à laquelle il sera fait référence un peu plus loin. M. Touzet a bien voulu nous autoriser à en faire état dans le présent article. 
successifs depuis la création de l'ONF montre la volonté constante du législateur de permettre la réalisation de ventes amiables. Lors de l'examen de la loi d'orientation sur la forêt de 2001, cet article, reflet d'un consensus ancien en faveur de l'ouverture à l'ONF de la possibilité de procéder à des ventes amiables, n'a pas fait l'objet de débats au fond. Tout au plus, un amendement de pure forme a permis, lors de la séance du 4 avril 2001 au Sénat, un bref commentaire du sénateur Philippe François, rapporteur, "les modes de vente de l'ONF sont ainsi adaptés en vue de permettre un recours plus large aux procédures de vente à l'amiable, susceptibles de lui garantir des débouchés plus réguliers ». Le sénateur Yann Gaillard, aujourd'hui président de la Fédération nationale des communes forestières, a pour sa part déclaré, évoquant les problèmes spécifiques des chablis de 1999 : "Notons au passage que les résultats obtenus l'ont été au prix d'un assouplissement considérable des méthodes de commercialisation des bois... C'était, en somme, anticiper sur votre projet de loi, qui prévoit que les méthodes de commercialisation des bois devront être plus diversifiées et mieux répondre aux situations dans le temps et dans l'espace ». Dans l'exposé des motifs du projet de loi, M. Glavany, ministre de l'Agriculture, précisait qu'il s'agissait de "favoriser la souplesse des conditions de ventes par l'ONF, aménagées pour augmenter la sécurité et la fluidité des approvisionnements des entreprises »(8).

Une modification des dispositions de l'article R.134-16 était cependant nécessaire du fait de la disparition de l'article L. 134-8 auquel il renvoie, mais il s'agit là d'un simple toilettage. De même au $4^{\circ}$ de l'article, le motif de vente amiable "Pour les chablis dans les coupes en exploitation" pourrait être étendu à tous les cas de chablis par simple suppression du membre de phrase "dans les coupes en exploitation».

Le rapport établi par M. le député Juillot (2003) au terme de la mission qui lui fut confiée par le Premier ministre le 6 décembre 2002 comporte des propositions de rédaction du décret d'application du texte relatif aux ventes amiables, ainsi “modifié" par la LOF du 9 juillet 2001. Ce rapport affiche une position de principe favorable à un large développement des ventes amiables dans le droit-fil des positions unanimement exprimées lors de l'examen de la loi. Mais à cette fin, tout en se référant à la disposition législative "nouvelle " introduite par la LOF sur les ventes amiables dont "le décret d'application, non pris à ce jour, a donné lieu à l'établissement d'un projet avec les ministères concernés à l'occasion de la mission ", il propose d'" organiser un marché de répartition négocié, qui coexisterait avec un dispositif d'appel à la concurrence... nécessitant la fixation de barèmes de prix ».

\section{LA LOI SUR LE DÉVELOPPEMENT DES TERRITOIRES RURAUX (LDTR) DU 23 FÉVRIER 2005}

Plusieurs facteurs vont alors se conjuguer pour conduire à une nouvelle modification de la loi.

\section{Problèmes posés par l'application de la loi du 9 juillet 2001}

Un projet de décret d'application des dispositions de la loi de 2001 avait été préparé par la Direction générale de la Forêt et des Affaires rurales (DGFAR). La rédaction proposée substituait deux articles à l'ancien R.134-16. Celui-ci devenait R.134-17, et un nouvel article R.134-16 aurait été constitué de quatre alinéas :

- le premier, rappelant la loi, donc non normatif, donc inutile,

- le second tentant de définir a priori la part de marché " faisant l'objet d'une offre globale de vente amiable ", et à cette fin introduisant au moins trois notions nouvelles, "massif »,

(8) Exposé des motifs de la loi d'orientation forestière, p. 6. 
" nature de coupe ", et " unité de marché ", susceptibles de poser de redoutables problèmes de définition, pour finir par renvoyer à « un pourcentage de référence par massif et par produits fixé par le Conseil d'administration",

- le troisième après avoir prévu le "porté à connaissance de l'offre de ventes amiable ", décrivant une procédure d'appel d'offres, et renvoyant à des clauses générales de ventes qui ne sont pas de nature réglementaire,

- enfin le quatrième décrivant un système de répartition dont l'apparition comme modalité de vente à l'amiable ne pouvait procéder que d'une erreur manifeste sur cette notion même.

Ce projet fut analysé dans le cadre du rapport que nous avions remis, en juin 2003, dans le cadre du CGGREF suite à une demande du directeur de Cabinet du Ministre sur "les fruitières $(9)$ de Franche-Comté et l'amélioration de la sécurité juridique des ventes de gré à gré réalisées par l'ONF ». Il ne respectait ni la lettre ni l'esprit de la modification des articles L.134-7 et L.134-8 apportée par la LOF. Rien n'éclairait l'ajout de motifs " économiques ». En outre, le corps du texte comportait toujours l'expression vente " amiable » et non pas le terme " gré à gré " qui venait de lui être substitué, confirmant l'absence de portée normative de cette modification. Surtout, la volonté du législateur a bien été de réitérer clairement le droit pour l'ONF de conclure des ventes amiables et de développer ce mode de vente. Il eut été paradoxal que la réitération en 2001 de cette volonté ancienne du législateur de faciliter les ventes amiables se traduise par un règlement complexe et réducteur qui n'existait pas avant. Le projet sortait du champ de la vente amiable telle que la définit le code civil, le résultat du libre accord des parties, puisqu'il introduisait une référence à des barèmes de prix fixés ex ante et prévoyait un dispositif de répartition qui n'a rien à voir avec la LOF votée. II aurait en outre présenté des risques tant vis-à-vis du droit de la concurrence, (nécessité d'une consultation préalable du Conseil de la concurrence), que du droit communautaire. Le rapport (Estrade et Morin, 2003) concluait donc :

"Ce nouvel article $R$ 134-16 est donc contraire à la volonté du législateur et lourd de problèmes juridiques. Nous ne pouvons que recommander d'écarter cette proposition ». Cette recommandation fut suivie par la DGFAR.

\section{De nouvelles propositions dans le cadre du projet de loi pour le développement des territoires ruraux}

Cependant, un document établi par la direction générale de l'ONF en date du 12 mars 2003 proposait de modifier encore ce cadre législatif fraîchement voté. Deux fiches avaient été établies à cette fin avec la Direction de l'Espace rural et de la Forêt (DERF) devenue DGFAR, en vue d'en proposer l'insertion dans le projet de LDTR. La principale proposition consistait en un article L.121-5 nouveau, constitué de quatre alinéas. L'idée originale, au deuxième alinéa, visait à donner à l'ONF "la capacité juridique à réaliser l'ensemble des transactions commerciales opérées en exécution de cette mission " (la mission générale de gestion confiée à l'ONF ; voir ci-après).

Cette proposition paraissait justement procéder du problème des fruitières de Franche-Comté. Est-ce que l'ONF avait la capacité juridique de les gérer ? L'article L.121-2 du Code forestier précise que l'ONF " est chargé de la gestion et de l'équipement des forêts... appartenant à l'État ». Se pose alors l'interprétation du mot " gestion ». Le Code rural, dans son article L.311-1, donne une définition de l'activité agricole, prise en compte par le Code général des Impôts (CGI) et appliquée de fait à la forêt privée, " activités dans le prolongement de l'acte de production et qui ont pour support l'exploitation ». Cette définition couvre la commercialisation

(9) Fruitière : coopérative (de fabrication des fromages ou du vin, ou de vente de bois) dans les régions avoisinantes de la Suisse (NDLR). 
des produits de la forêt. Elle s'applique donc aux forêts relevant du régime forestier, par le jeu de l'article L.144-1 du Code forestier (Nota : le Code rural définit l'espace rural comme agricole et forestier).

Enfin, l'article L.121-4, réécrit sans changement au fond, par l'article 50 de la LOF prévoit que "l'ONF peut être chargé par voie de convention avec des personnes publiques ou privées d'opérations de gestion... en vue de l'aménagement et du développement durable des ressources forestières ». Les dispositions réglementaires prises pour son application, l'article R.121-6 modifié par le décret du 22 juin 2003, ne prévoient pas explicitement les opérations de commercialisation, alors même que ledit décret comporte la création d'un article R.121-6-1 nouveau, qui définit clairement le rôle de l'ONF intervenant comme mandataire d'une ou plusieurs personnes publiques, ce qui correspond exactement au problème des fruitières de Franche-Comté.

L'examen de l'arrêt CE 91549, déjà cité, relatif au contrat de Sougy, apporte en fait la solution du problème. En effet, un de ses considérants confirme qu'un contrat de commercialisation est bien un acte de gestion d'un domaine forestier : "le litige dont ce tribunal (le tribunal administratif de Paris) était saisi ne concernait pas l'activité de protection, de conservation et de surveillance de la forêt qui relève de la mission de service public administratif dévolue à l'office ; qu'il n'était pas détachable de son activité de service public industriel et commercial chargé de la gestion du domaine forestier et de l'équipement des forêts ; que dans ces conditions, c'est à bon droit que les premiers juges ont estimé que la demande du groupement ressortissait à la compétence des juridictions de l'ordre judiciaire ». Le problème posé pouvait donc être résolu par voie de convention, dans le cadre des textes existants, aussi ces propositions de modification ont-elles été écartées du projet de loi sur le développement des territoires ruraux.

\section{La sécurité juridique des agents de l’ONF}

L'ONF avait par ailleurs insisté sur le problème de la sécurité juridique de ses agents, constatant à juste titre l'inquiétude suscitée chez ses personnels par la mise en examen d'un directeur régional, de son adjoint et d'un chef de service départemental, pour "vente clandestine" après avoir conclu sous forme de marché de gré à gré un avenant à un contrat originellement mis en appel d'offres.

Bien entendu, l'ONF a assuré la défense des agents mis en cause. L'affaire s'est terminée par un non-lieu, confirmé le 12 mars 2003 par un arrêt de la chambre criminelle de la Cour de cassation. Cependant, le non-lieu repose sur un point de procédure; aussi subsistait-il un doute sur le fond de l'affaire qui pouvait se représenter pour d'autres agents et dont l'impact psychologique pouvait freiner le développement des marchés de gré à gré et des formules contractuelles.

Une note du cabinet d'avocat chargé de la défense de l'ONF estimait qu'un avenant est régulier au regard des règles des marchés publics " dès lors qu'il n'a pas pour objet le bouleversement de l'économie du contrat ", et qu'en pratique "le Conseil d'État admet en matière de marchés publics une règle de $10 \%$ ». L'article 2 du contrat en cause encadrait la possibilité d'avenants dans une fourchette de plus ou moins $20 \%$. L'ONF considérait cette marge nécessaire, compte tenu de la spécificité des ventes de bois sur pied. Une solution pouvait être alors de prévoir dans les modifications à apporter au R.134-16 une telle marge en matière d'avenants passés de gré à gré. Cette note considérait qu'il y avait problème au regard des articles L.134-7 et L.134-8, et proposait afin de les régler "d'élaborer un mécanisme contractuel permettant d'éviter ces différents écueils ».

Une modification législative s'avérait donc nécessaire. 


\section{La clarification apportée par la LDTR}

De toutes les façons, nous l'avons vu, la LOF de 2001 posait un problème de coordination rédactionnelle, puisque l'article L.134-1 qui fixe le cadre légal des ventes vise l'article L.134-8 que la LOF vient de supprimer. Dans ces conditions, il est apparu préférable de profiter de cette nécessité de clarification pour introduire une nouvelle rédaction simplifiée mettant les deux modes de vente sur le même plan. Cette solution simple avait le mérite de prévenir un débat qui risquait de s'instaurer sur la notion de vente clandestine à partir de l'article L.134-1, aussi fut-elle retenue par l'ingénieur général Drège qui venait de prendre les fonctions de directeur général de l'ONF dans un contexte financier et social difficile. La nouvelle équipe dirigeante de l'ONF prit très vite la mesure de l'importance du cadre juridique des ventes pour le devenir de l'établissement.

La formulation introduite dans le projet de LDTR, intégralement déjà citée en tête de cet article, précise que : "Les coupes et produits des coupes dans les bois et forêts de l'État sont vendues par l'Office national des forêts soit par adjudication ou appel d'offres, soit de gré à gré, dans les conditions fixées par décret en Conseil d’État». Les deux modes de vente sont désormais mis sur le même plan, ce qui écarte la notion de vente clandestine. En introduisant, dans le décret, la référence à une procédure interne d'approbation, on évite des exégèses du Code forestier conduisant à interpréter comme vente clandestine des actes de gestion courante dont le législateur souhaite le développement. C'est donc ce qui fut fait à l'article 2 du décret du 23 novembre 2005 reproduit ci-dessous :

«VIII. - L'article R.* 134-15 est précédé de l'intitulé suivant :

"Sous-section 4 "

"Ventes de gré à gré "

IX. - L'article R.* 134-15 est remplacé par les dispositions suivantes :

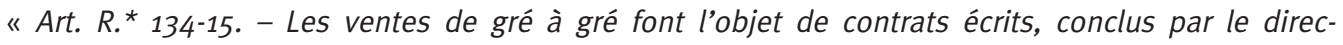
teur général de l'Office national des Forêts ou son délégataire. Elles donnent lieu soit à des contrats d'approvisionnement à exécution ou à livraisons successives et pour une durée ne pouvant excéder cinq années, soit à des ventes à exécution ou à livraison immédiate lorsque les produits sont disponibles à la vente».

L'examen par le Conseil d'État a conduit à compléter le texte initial du Gouvernement par la deuxième phrase. En l'absence de débat lors de l'adoption du texte, le ministre n'avait pas eu l'opportunité de s'expliquer sur ses intentions, ce qui fait que la question a pu se poser devant le Conseil d’État de la portée d'une phrase du rapport du sénateur Émorine, élu de Saône-etLoire comme le député Juillot. Cette phrase figure à la page 368 du rapport de la Commission des affaires économiques du Sénat : "la modification proposée pour l'article L.134-7 est plus substantielle, puisque :

- elle renvoie désormais aux techniques de vente des bois par l'ONF, en lieu de leur procédure, ce qui doit être un élément de clarification du texte ;

- elle facilite le recours à la technique de la vente de gré à gré, plus souple et surtout seule adaptée dans certaines situations où il n'y a que quelques acheteurs potentiels, voire un seul. La rédaction proposée remplace, pour le recours à la vente de gré à gré, la condition de " motifs d'ordre technique, commercial ou économique » définis par décret en Conseil d'État, par la notion plus générale de "conditions fixées par décret en Conseil d’État " ".

Certains voulurent y voir la justification d'une introduction dans le décret des propositions du rapport Juillot écartées par le Gouvernement, comme on l'a vu plus haut. Après en avoir délibéré, la section des Travaux publics a validé le texte du Gouvernement, dans l'esprit de la volonté explicite et réitérée du législateur. 
Dès l'Ordonnance de 1669 , le législateur a voulu réglementer les coupes dans le double but d'éviter la surexploitation de la forêt, en posant le principe d'un règlement de coupes, et de contrôler les modalités de leur vente par les agents de l'État, reprenant en cela des textes antérieurs. Dans le contexte de l'époque, ceux-ci étaient soumis à des tentations d'autant plus fortes que, du fait des distances et de leur isolement, les possibilités de contrôle étaient faibles.

La vente du bois, donc sa commercialisation et son extraction de la forêt, constitue le terme du processus de gestion forestière nécessaire à la pérennité du cycle de la forêt. Ce point devient essentiel au moment où on commence à prendre conscience des conséquences à long terme de la sous-exploitation de la forêt française dont le taux d'exploitation globale est au mieux de l'ordre de $58 \%{ }^{(10)}$ (le fait qu'il soit sensiblement meilleur dans les forêts publiques a pour corollaire qu'il est encore plus faible en forêt privée, à l'exception du massif des Landes de Gascogne). De ce fait, dans de nombreuses régions, la pérennité à long terme des forêts est en cause. Au regard de cet impératif sylvicole, les modes de vente ne doivent pas constituer un goulot d'étranglement. Les modalités de contrôle et de vente ont largement évolué en fonction du contexte général de la société française et de la mise en place d'une administration rigoureuse et audessus de tout soupçon.

Dès le début du XXe siècle, la possibilité de ventes de gré à gré a été introduite dans le Code forestier. Mais qu'il ait fallu quatre-vingts ans entre une première légalisation dérogatoire et leur "banalisation" est révélateur des intérêts sous-jacents au maintien du dispositif antérieur. Pendant longtemps des scieurs et des industriels étaient pourtant demandeurs de contrats de gré à gré. Mais certains acheteurs de bois ont à l'évidence intérêt au maintien du système traditionnel auquel ils se sont parfaitement adaptés pour leur plus grand avantage immédiat, même si à terme il en résulte un frein indirect à leur développement et à l'industrialisation du secteur. Parallèlement, il existait de fortes réticences au sein de l'ONF, comme l'ont montré les controverses autour du projet de Sougy pendant les années $1980^{(11)}$. La façon dont n'a pas été mis à profit l'arrêt du Conseil d'État validant le contrat de Sougy est éclairante. Le changement est grand pour les gestionnaires qui vont cesser d'être de simples producteurs et qui vont devoir désormais s'impliquer complètement dans tous les aspects de la commercialisation des bois.

Alfred ESTRADE - Georges-André MORIN

Ingénieurs généraux du Génie rural, des Eaux et des Forêts

CONSEIL GÉNÉRAL DU GREF

251, rue de Vaugirard

F-75732 PARIS CEDEX 15

(alfred.estrade@agriculture.gouv.fr)

(georges-andre.morin@agriculture.gouv.fr)

\footnotetext{
(10) «Indicateur de résultat », publié par le ministère et cité par le DGFAR, au colloque annuel du "club des EPN" (établissements publics nationaux) le 22 juin 2004 .

(11) Ce que confirment des transmissions de documents entre l'ONF et la Fédération nationale du Bois, tant pendant la négociation du contrat qu'au moment où s'est noué le contentieux, figurant dans le dossier du contentieux de Sougy que nous avons consulté lors de notre mission de 2003.
} 


\section{BIBLIOGRAPHIE}

BÉTOLAUD (Y.), MÉO (J.). - La Filière-bois. - Paris : La Documentation française, 1980.

COIN-DELISLE (-), FREDERICH (-). - Commentaire sur le Code forestier. - Paris : Pélicier et Chatelet libraires, 1827/1828. (Exposés des motifs et rapports de présentation devant la Chambre des Députés et devant la Chambre des Pairs, ensemble des articles commentés, et ordonnance d'application du $1^{\text {er }}$ août 1827).

DELABALLE (Ch.). - Le Sentiment d'entreprendre. Ce recueil inédit des souvenirs du premier directeur général de l'Office national des Forêts est accessible à la bibliothèque du centre de Nancy de l'ENGREF.

ESTRADE (A.), MORIN (G.-A.). - Les fruitières de Franche-Comté et l'amélioration de la sécurité juridique des ventes de gré à gré réalisées par l'ONF. Rapport du CGGREF, remis fin juillet 2003 et présenté en $4^{\mathrm{e}}$ section le 3 septembre 2003 .

MEYER (F.). - Législation et politique forestières. - Éditions Berger-Levrault, 1968.

ORIEUX (J.). - Jean de La Fontaine. Biographie. - Paris : Flammarion, 1976.

L'Ordonnance des eaux et forêts du mois d'août 1669. - Paris : Debure père (quai des Augustins), 1775.

RONDONNEAU (L.). - Code forestier ou recueil des Lois, Arrêtés, Décrets et avis du conseil d’État relatifs à l'établissement et à l'organisation de l'administration forestière et au Régime des Bois et Forêts de l'État, des Communes, des Établissements publics et des particuliers, depuis l'an IX, jusqu'à ce jour. - Paris, juillet 1810 .

JUILLOT (D.). - La Filière-bois française : la compétitivité, enjeu du développement durable. Rapport au Premier ministre. - Revue forestière française, vol. LV, $\mathrm{n}^{\circ}$ 3, 2003, pp. 185-296.

Les autres références se trouvent dans les Journaux officiels des débats et dans les rapports des assemblées parlementaires.

HISTORIOUE DE L'ÉULLUTION DU CADRE LÉgISLRTIF ET RÉGLEMENTRIRE DES MODES DE UENTES DES BOIS DES FORÊTS PUBLIOUES [Résumé]

Le cadre juridique des ventes des forêts publiques est resté intangible pendant trois siècles, de l'Ordonnance de 1669 à la loi de Finances rectificative du 24 décembre 1969. Seule l'adjudication publique était possible à l'exclusion de toute autre modalité. Certes, dès 1926, un dispositif dérogatoire de ventes amiables a été introduit permettant de faire face à des situations particulières. Dans le prolongement de la réforme créant l'ONF, le premier directeur général de l'établissement obtenait en 1969 un élargissement substantiel du champ des ventes amiables. Cependant, la mise en œuvre de ces nouvelles possibilités s'est durablement heurtée aux conservatismes et aux intérêts acquis, bien que le dispositif ait été ensuite par deux fois confirmé par le législateur. La quatrième et dernière modification, apportée par la loi de Développement des territoires ruraux, en sortant du cadre dérogatoire et en mettant les deux modes, adjudication et vente amiable, sur le même plan lève les derniers obstacles juridiques.

\section{HISTORICAL BACKGROUND OF CHANGES IN THE LEGISLATIUE AND REGULATORY FRAMEUORK FOR SALES OF STATE FOREST TImBER [Abstract]}

For three centuries, the legal framework for selling timber from state forests remained unchanged, i.e. from the 1669 Ordinance to the Amending Appropriation Bill of December 24, 1969. Public auction was the only allowable sales channel, although as early as 1926, a waiver allowing private sales was introduced to deal with specific situations. In the wake of the reform that established the ONF (French Forestry Board), the scope of private sales was substantially broadened in 1969 at the instigation of its first Director General. However, the implementation of these new possibilities came up against conservatism and vested interests, in spite of the provisions being confirmed by lawmakers twice thereafter. The fourth and most recent amendment, brought in by the Bill on Development of Rural Areas, brought the issue outside of the scheme of waivers and put the two modes, public auction and private sale, on an equal footing thereby removing the remaining legal obstacles. 